\title{
COOL CARBON STARS IN OPEN CLUSTERS
}

\author{
A. ALKSNIS and Z. ALKSNE
}

Radioastrophysical Observatory, Latvian Academy of Sciences, Latvia, U.S.S.R.

\begin{abstract}
By combining lists of carbon stars with the catalogue of open clusters (Alter et al., 1970), a list of variable carbon stars, being probable members of clusters is given. The number of stars is too small to derive reliable statistics on cluster membership. Too few radial velocities are yet available. Photometric criteria are still too inaccurately known.

Absolute magnitudes in the infrared region $(\lambda=1.04 \mu)$ were recently derived by Baumert (1974).
\end{abstract}

TABLE I

Variable carbon stars near open clusters

\begin{tabular}{|c|c|c|c|c|}
\hline $\begin{array}{l}\text { No. } \\
\text { GCCCS }\end{array}$ & $\begin{array}{l}\text { Star } \\
\text { designation }\end{array}$ & Cluster & $\begin{array}{l}Q / r \\
\text { relative } \\
\text { angular } \\
\text { distance }\end{array}$ & $\begin{array}{l}V- \\
\text { magnitude }\end{array}$ \\
\hline 252 & HN Aur & NGC 1664 & 0.2 & $11^{m} 2-11^{m} 6$ \\
\hline 1275 & & NGC 2660 & 0.3 & \\
\hline 3187 & V 433 Cas & Czernik 43 & 0.5 & \\
\hline 3187 & V 433 Cas & NGC 7654 & 2.8 & \\
\hline 86 & EW Per & Stock 4 & 0.6 & \\
\hline 538 & & Collinder 106 & 1.0 & $12.8-13.3$ \\
\hline 148 & BI Per & Berkeley 9 & 1.1 & \\
\hline 1604 & SZ Car & NGC 3114 & 1.2 & \\
\hline 2724 & U Lyr & NGC 6791 & 1.3 & \\
\hline 2870 & AY Cyg & Dolidze 2 & 1.7 & \\
\hline 2873 & RY Cyg & NGC 6883 & 1.7 & $9.0-9.3$ \\
\hline 2851 & SVS 1633 & Dolidze 37 & 1.8 & $12.4-14.4$ \\
\hline 2519 & V $1948 \mathrm{Sgr}$ & NGC 6541 & 1.9 & \\
\hline 2883 & V432 Cyg & Dolidze 3 & 1.9 & $10.5-11.1$ \\
\hline 112 & VZ Per & Trumpler 2 & 1.9 & \\
\hline 2874 & V 429 Cyg & NGC 6883 & 2.0 & $10.6-11.4$ \\
\hline 339 & & NGC 1912 & 2.3 & $14.5-15.1$ \\
\hline 1750 & TZ Car & IC 2606 & 2.3 & \\
\hline 3210 & SVS 1730 & NGC 7789 & 2.4 & $9.6-10.9$ \\
\hline 107 & DY Per & Trumpler 2 & 2.4 & \\
\hline 814 & BF Pup & NGC 2422 & 2.8 & \\
\hline 2756 & AR Vul & Stock 1 & 2.7 & \\
\hline 338 & & Stock 8 & 2.8 & $13.3-15.5$ \\
\hline - & SVS 1731 & NGC 7789 & 3.0 & $12.0-14.0$ \\
\hline
\end{tabular}

\section{References}

Alter, G., Ruprecht, J., and Vanýsek, V.: 1970, Catalogue of Star Clusters, 2nd ed., Budapest. Baumert, J. H.: 1974, Astrophys. J. 190, 85. 